\title{
La participación de los lectores en la prensa alternativa: análisis histórico de cuatro revistas catalanas
}

\author{
Readers' participation in the alternative press: historical analysis of our
}

Catalan magazines

\author{
Camps-Durban, Eloi \\ Universitat Pompeu Fabra (UPF) \\ eloi.camps01@estudiant.upf.edu
}

Forma de citar este artículo:

Camps-Durban, E. (2020). “La participación de los lectores en la prensa alternativa: análisis histórico de cuatro revistas catalanas", RAEIC, Revista de la Asociación Española de Investigación de la Comunicación, vol. 7, núm. 13, 140-163

https://doi.org/10.24137/raeic.7.13.7

\section{Resumen:}

Las audiencias activas son un actor cada vez más relevante en el ecosistema comunicativo digital. Sin embargo, la participación en los medios no es un fenómeno reciente, sino que presenta una larga historia. Estudiar su desarrollo es útil para comprender las actuales fórmulas de interacción entre medios y públicos y la diversidad de sus enfoques. En particular, la participación es un elemento clave de los medios alternativos, con una estructura y organización horizontales más abiertas a la implicación de la sociedad civil que los medios convencionales. Este artículo ofrece una 
visión histórica de la participación en los medios alternativos a partir del análisis de cuatro revistas de Cataluña, un territorio con una rica tradición de prensa alternativa. Se clasifican y analizan las oportunidades que tienen los lectores de publicar contenidos, incidir en la configuración de la agenda y participar en la toma de decisiones. Como objetivo secundario, el artículo bosqueja una cronología para una historia de la comunicación alternativa catalana.

Palabras clave: audiencias activas, participación, compromiso, medios alternativos, prensa alternativa, prensa catalana.

\begin{abstract}
:
Active audiences are an increasingly relevant actor in the digital communication ecosystem. However, media participation is not a recent phenomenon, but one that presents a long history. Studying its development is useful to comprehend the current formula of interaction between media and publics and the variety of their approaches. In particular, participation is a key feature of alternative media, which hold horizontal structures and modes of organization that are more open to the engagement of civil society than mainstream media. This paper offers an historical vision of participation in alternative media based on the analysis of four magazines located in Catalonia, a region with a rich alternative press tradition. The opportunities for the readers to publish content, influence the agenda-setting and participating in decision-making are classified and analyzed. As a secondary objective, this paper sketches a chronology for a Catalan alternative communication history.
\end{abstract}

Keywords: active audiences, participation, engagement, alternative media, alternative press, Catalan press.

\title{
1. INTRODUCCIÓN
}

En el ecosistema mediático digital las audiencias ganan protagonismo y tienen la capacidad de intervenir en los procesos de producción y distribución de la información 
(Masip, Guallar, Peralta, Ruiz y Suau, 2015). La participación es un elemento básico de cualquier medio de comunicación contemporáneo y altera la relación vertical y unilateral entre periodistas y públicos propia de la sociedad analógica (Paulussen, Heinonen, Domingo y Quandt, 2007). Las audiencias activas, pues, obligan los medios a tener en cuenta las formas de interacción que, desde el advenimiento de la web 2.0, permiten a cualquier usuario producir y diseminar contenidos por la red con gran facilidad. Por otro lado, las vías abiertas para la participación en el entorno digital han supuesto el incremento e innovación en los sistemas de rendición de cuentas de los medios (Mauri-Ríos y Ramon-Vegas, 2015).

Entre los enfoques y estrategias de participación, los que ponen en práctica los medios de comunicación alternativos merecen una especial atención por su filosofía participativa (Bailey, Cammaerts y Carpentier, 2007; Carpentier, Dahlgren y Pasquali, 2014) y el interés académico que han despertado en las últimas dos décadas (Harcup, 2011; Jeppesen, 2016). La generalización de Internet ha supuesto la multiplicación de iniciativas de comunicación alternativa, aunque la historia de los medios alternativos corre paralela al desarrollo del periodismo en la modernidad (Atton y Hamilton, 2008; Forde, 2011). Desde su aparición, han estado en la vanguardia del periodismo participativo; por eso constituyen un objeto de análisis significativo para entender la evolución de los mecanismos de interacción que posibilitan las audiencias activas en los medios de comunicación.

El presente artículo tiene por objetivo analizar los mecanismos de participación en la prensa alternativa de los siglos XX y XXI. La investigación focaliza en Cataluña, que ha tenido históricamente un papel notable en la comunicación alternativa en el Estado español (Sáez, 2009, p.151). Se parte de la hipótesis que la prensa alternativa ha evolucionado en lo referente a herramientas y canales de participación, los cuales han aumentado progresivamente. A través del análisis de cuatro publicaciones de épocas distintas, se observará si existe diversidad de modelos participativos y cuál ha sido su adaptación a los cambios de contexto sociopolítico, económico y comunicativo. Mientras que en la investigación anglosajona existen obras sobre la evolución histórica de la participación en los medios (Griffen-Foley, 2004; Carpentier, Dahlgren y Pasquali, 
2014) y específicamente en los alternativos (Atton y Hamilton, 2008), éste es un tema todavía inédito en el contexto español. Como objetivo secundario, la presente investigación pretende realizar una contribución para una historia de la prensa alternativa catalana, una de las diversas lagunas en los estudios de este ecosistema comunicativo (Guillamet, 2003).

Entre los enfoques existentes en teoría y análisis de la comunicación alternativa, adoptamos el de los medios críticos (Fuchs, 2010) y radicales (Downing, 2001). Estos dos modelos comparten características (Jeppesen, 2016) y son los que, en el contexto catalán, presentan una historia más larga, hecho que permite una comparativa histórica de más alcance. Por el mismo motivo, focalizaremos en la prensa escrita, puesto que es el soporte con más tradición y que constituye uno de los fenómenos más investigados dentro de los estudios en comunicación alternativa. El alcance cronológico del estudio se divide en cuatro periodos: el primer tercio del siglo XX, el franquismo y la transición, la etapa democrática y el escenario poscrisis de 2008. Los tres primeros son identificados por Tresserras (1999) como tres etapas en las que se configuran los sistemas de comunicación y cultura en Cataluña, en estrecha relación con la realidad política, social y económica del país. Respecto al cuarto periodo, todavía en curso, está marcado por el estancamiento de los sistemas democráticos, la crisis de los medios y del periodismo y las nuevas formas de comunicación digital (Carpentier, Dahlgren y Pasquali, 2014, p.135). Se trata, pues, de cuatro escenarios mediáticos con una dinámica propia, que influye en los medios alternativos y sus posibilidades de participación.

\section{MARCO TEÓRICO}

\subsection{PARTICIPACIÓN Y MEDIOS DE COMUNICACIÓN}

La participación es la situación en que los actores implicados en la toma de decisiones están posicionados unos respecto a otros a través de relaciones de poder, y que requiere oportunidades estructurales - mecanismos institucionalmente habilitados para participar - para producirse (Carpentier, Dahlgren y Pasquali, 2014, p.124-126). Aplicada a los medios de comunicación, consiste en la intervención de los receptores en relación a los contenidos mediáticos y en la toma de decisiones organizativas; esta 
manera de hacer periodismo es generalmente conocida como periodismo participativo (Suau y Masip, 2011). La participación de los públicos no es una característica exclusiva de los medios digitales, sino que es un fenómeno que tiene, al menos, un siglo y medio de existencia (Griffen-Foley, 2004; López-García, 2007). Sin embargo, las tecnologías digitales y los entornos transforman e incrementan las posibilidades de interacción de los públicos hasta al punto de obligar a repensar los roles de periodistas y lectores (CruzÁlvarez y Suárez-Villegas, 2012).

Schultz define tres modelos de relación entre audiencia y medios, aplicable tanto a los analógicos como a los digitales, según el grado de reactividad: la comunicación unilateral, en que no hay interacción entre los mensajes - cartas y artículos de los lectores-; la comunicación bilateral o reactiva, en que el mensaje obtiene respuesta, pero sin más continuidad - cartas a los redactores o al director que obtienen respuesta, encuestas, secciones de preguntas y respuestas, llamadas, comentarios en artículos- y la comunicación interactiva, en la cual se establece un diálogo donde se intercambian los papeles de emisor y receptor -encuentros con periodistas, mesas de discusión públicas, intercambios de correspondencia impresa o digital- (Schultz, 2000, pp.210211).

Más recientemente, Masip y Suau (2014) establecen otra triple distinción de fórmulas participativas, a partir del estudio de Rost (2006) sobre la interactividad del diario digital. La interactividad selectiva, apuntan los autores, implica la selección y personalización de la oferta de contenidos y servicios del medio; en la participativa, se produce interacción con los contenidos a través de mecanismos como comentarios, votaciones y valoraciones, que propiamente no aportan contenido nueva; finalmente, la interactividad comunicativa o productiva supone que los medios difundan contenidos creados por la audiencia. Relacionando esta clasificación con la de Schultz, podemos considerar que las herramientas de interactividad participativa responden a la comunicación bilateral e interactiva -el medio busca entablar un diálogo con el público-, mientras que la interactividad comunicativa se identificaría con la comunicación unilateral -el medio difunde contenidos enviados por el público, pero sin interactuar con ellos-. 
No obstante, los ideales normativos sobre participación contrastan con la realidad de sus fórmulas y sus resultados. El enfoque economicista de la participación, la reticencia de los profesionales a modificar las rutinas y a ceder su rol de gatekeepers y productores, la baja participación productiva de las audiencias y la falta de una estrategia participativa bien definida se perfilan como las causas del impacto limitado de la participación en los medios digitales (Masip, 2016; Appelgren y Salaverría, 2019). Por su lado, los medios alternativos históricamente han planteado una relación más participativa con sus audiencias (Barnes, 2014; Carpentier, Dahlgren y Pasquali, 2014) y desde una perspectiva no mercantilista.

\subsection{LOS MEDIOS DE COMUNICACIÓN ALTERNATIVOS}

Como señalan Harcup (2011) o Jeppesen (2016), entre otros, los medios alternativos han despertado entre los investigadores a partir de los 2000 un renovado y sostenido interés que ha contribuido a aumentar el conocimiento sobre la multiplicidad de experiencias de este ámbito comunicativo y a dinamizar la reflexión teórica. Bajo el término medios alternativos se agrupa un conjunto de proyectos comunicativos con planteamientos, estrategias y relaciones con otros medios de comunicación que presenta una diversidad muy notable, expresada en el modelo periodístico, la distribución de los contenidos, la participación, la organización interna y la financiación (Bailey, Cammaerts y Carpentier, 2007). Esta circunstancia dificulta la concreción de una definición general del concepto y genera un debate de carácter inacabado y dinámico (Fleischman, Reguero y Sáez, 2009).

El presente estudio se basa en la noción de medios alternativos entendidos como iniciativas que quieren transformar los sistemas de la comunicación y el periodismo hegemónicos (Atton y Hamilton, 2008; Tamarit, 2012), al mismo tiempo que incorporan un horizonte de transformación social global (Atton, 2002). Se pueden identificar dos grandes corrientes de investigación y teorización sobre los medios alternativos: la subjetivista, que engloba categorías como participativo, ciudadano y comunitario, y que enfatiza los actores y la participación; y la objetivista, que comprende categorías como 
radical y crítico, más centrada en las estructuras y los contenidos (Fuchs, 2010). Para este artículo, enmarcaremos el análisis en el paradigma objetivista.

Según estudios publicados (Sandoval, 2009; Sandoval y Fuchs, 2010; Fuchs, 2010), los medios críticos pretenden publicar "productos mediáticos de alta calidad" y "ganar visibilidad pública" (Sandoval, 2010, pp.12-13) para poder cuestionar las formas de dominación y plantear alternativas al sistema capitalista. Para alcanzar este objetivo, requieren una estructura de cierta envergadura y una dedicación profesionalizada, así como el acceso a redes de distribución que permitan llegar a públicos más amplios (Atton y Hamilton, 2008, pp.46). Esta opción, pese a que les ofrece más impacto, también les limita las posibilidades de participación (Hamilton, 2000). Sin embargo, el conjunto de medios alternativos se fundamenta en una ética participativa para propiciar la ciudadanía activa, entendida como la intervención tanto en los contenidos como en la toma de decisiones (Harcup, 2011). Por lo tanto, los medios críticos no renuncian a la participación ni a una organización abierta, aunque no hagan de ello su principal razón de ser.

Los medios radicales se inspiran en la ideología y prácticas del anarquismo (Jeppesen, 2016) y son, generalmente, no profesionales (Downing, 2001). Esta circunstancia difumina las barreras entre emisores y receptores y propicia, junto con la horizontalidad y la democracia interna, la participación de individuos y colectivos. El auge de los medios radicales, vinculados a las formas de acción política de los nuevos movimientos sociales, se produce en las décadas de los sesenta y setenta del siglo XX, y es uno de los factores que explica el fortalecimiento de la noción de participación en los proyectos mediáticos (Carpentier, Dahlgren y Pasquali, 2014).

\subsection{MEDIOS ALTERNATIVOS EN CATALUÑA}

La historia de los medios y del periodismo en Cataluña ha dedicado una atención todavía escasa a los medios alternativos. Falta una visión histórica global del fenómeno que defina sus etapas, características y evoluciones. Sin embargo, se dispone de algunos estudios de caso o sectoriales, como se detallará a lo largo de este epígrafe. Aunque este propósito excede las posibilidades del presente artículo, realizaremos un esbozo 
cronológico de diversos tipos de medios alternativos que han existido en el ecosistema mediático catalán para enmarcar nuestro análisis.

Se puede encontrar un precedente de los medios alternativos en las revistas satíricas, aparecidas durante la Renaixença como un modelo de medio dirigido a las clases populares y crítico con la prensa burguesa (Guillamet, 1994). A partir de 1868, la prensa obrera - con preponderancia de la anarquista - fue definiendo un primer modelo de prensa crítica (Dalmases, 1992). Aunque al principio se trataba de periódicos modestos dedicados a la divulgación doctrinal y sindical, algunos incorporaron progresivamente contenidos informativos y consiguieron profesionalizarse (Tavera, 1978, 1992), paralelamente a la transformación de la prensa diaria burguesa hacia modelos informativos y empresariales durante el primer tercio del siglo XX (Gómez-Mompart, 1992). También en esta época se desarrolla la prensa comarcal, modelo genuino catalán de periodismo local popular, no profesional y participativo (Guillamet, 1983), con puntos en común con la comunicación alternativa.

La Guerra Civil supone un corte en el desarrollo del sistema mediático catalán en su conjunto. La relativa apertura informativa y de difusión que estableció la Ley de prensa de 1966 fue aprovechada por las publicaciones de barrio de Barcelona (López, 1994) y por la prensa comarcal para ejercer un papel contrainformativo a partir del impulso popular y la organización horizontal. También en las postrimerías del franquismo surgieron intentos de una prensa clandestina alternativa profesionalizada (Vinyoles, Torns y Lanao, 1989).

El escenario abierto durante la transición a la democracia supuso la multiplicación y diversificación de la comunicación alternativa con las radios libres (Prado, 1980) y las televisiones comunitarias (Moragas y Prado, 2002). Asimismo, se inició la contracción de la prensa de barrio, consecuencia directa de la pérdida de protagonismo político del movimiento vecinal (López, 1994), mientras se asistía a la profesionalización de una parte de las cabeceras comarcales (Guillamet, 1983). La prensa alternativa continuó desarrollándose en vinculación más o menos estrecha con movimientos sociales como 
el okupa (González, 2011, pp.221-225), el ecologista y el feminista, de manera que el modelo más abundante era el de prensa radical.

A finales de los noventa se produce un cierto estancamiento de los medios alternativos, fruto del reflujo de los movimientos sociales (Tubau y Vallès, 1995, pp.15). No obstante, la generalización de Internet ofrece, a partir de los 2000, nuevas posibilidades para los medios alternativos. En episodios de activación de la protesta ciudadana, los medios alternativos se ven favorecidos; fue el caso de las olas de movilizaciones del $15 \mathrm{M}$ en el año 2011 (Barranquero y Meda, 2015). Por otro lado, la conjuntura política, social y económica abierta por la crisis de 2008 ha supuesto un estímulo para nuevos proyectos de periodismo alternativo que, en forma de asociación o cooperativa, han nacido en los últimos diez años en Cataluña y han revitalizado el panorama de medios críticos (Barranquero y Sánchez, 2018).

\section{METODOLOGÍA}

En relación a la muestra, se ha realizado un muestreo no probabilístico por conveniencia (Mejía, 2000, pp.169) para seleccionar una cabecera de prensa alternativa de cada uno de los periodos analizados. Esta fórmula responde al carácter pionero de este estudio y sus dimensiones, así como a la falta de disponibilidad de datos cuantitativos sobre la mayoría de medios alternativos catalanes, hecho que dificulta establecer parámetros de selección más objetivos. Se parte de dos criterios básicos: se tiene que tratar de cabeceras de prensa alternativa que encajen en el paradigma de medios críticos y/o radicales y que tengan un alcance del conjunto del territorio catalán como mínimo. Para asegurar su relevancia, se han escogido revistas que hayan sido estudiadas previamente, o bien que presenten una trayectoria suficientemente larga que avale su consolidación. Los cuatro casos que forman la muestra de la investigación son La Revista Blanca (19231936), API (1972-1976), Cruïlla - Illacrua (1992-2009) y Directa (2016).

Se ha realizado una aproximación cuantitativa a partir del análisis de contenido de cada revista, para detectar los mecanismos de participación que ofrecen y clasificarlos según las tipologías de interacción planteadas por Schultz (2000), incorporando el matiz de 
Masip y Suau (2014) en la distinción entre interacción participativa y productiva, mencionadas en el epígrafe 2.

Tabla 1. Tipos de interacción y mecanismos.

\begin{tabular}{|c|l|}
\hline Tipo de interacción & \multicolumn{1}{c|}{ Mecanismos } \\
\hline Unilateral & $\begin{array}{l}\text { Contenidos enviados por los lectores (interactividad } \\
\text { productiva) } \\
\text { Cartas al director o a los redactores (interactividad productiva) }\end{array}$ \\
\hline \multirow{5}{*}{ Bilateral } & $\begin{array}{l}\text { Encuestas y votaciones (interactividad participativa) } \\
\text { Sección de preguntas y respuestas (interactividad } \\
\text { participativa) } \\
\text { Comentarios a los artículos (interactividad participativa) } \\
\text { Correspondencia con la redacción (interactividad participativa) } \\
\text { Réplicas de los lectores (interactividad productiva) }\end{array}$ \\
\hline \multirow{3}{*}{ Comunicativa } & $\begin{array}{l}\text { Correspondencia con la redacción (interactividad participativa) } \\
\text { Reuniones y encuentros con el director o los redactores } \\
\text { (interactividad participativa) } \\
\text { Actos públicos, fórums y otros espacios de debate (interactividad } \\
\text { participativa) }\end{array}$ \\
\hline
\end{tabular}

Fuente: Elaboración propia a partir de Schultz (2000, p. 211) y Masip y Suau (2014).

Esta observación y clasificación se refuerza y contrasta, en el caso de La Revista Blanca y $A P I$, con la consulta de estudios monográficos sobre estas publicaciones, mientras que para Cruilla - Illacrua y Directa se han realizado entrevistas semiestructuradas a miembros de cada revista, centradas en las vías de participación y relación con la audiencia $^{1}$. De esta manera, obtenemos datos cualitativos sobre el enfoque de la participación de estos medios y, en especial, sobre si contemplan algún mecanismo de comunicación interactiva.

\section{ANÁLISIS}

\subsection{LA REVISTA BLANCA}

Esta revista es considerada la máxima representante de las publicaciones anarquistas independientes; es decir, aquellas que no dependían de ningún sindicato y que

\footnotetext{
${ }^{1}$ Los informantes son Jordi Garcia, de Cruïlla - Illacrua (entrevista realizada telefónicamente el 17 de septiembre de 2019) y Ester Mora, de Directa (entrevista realizada en la sede del medio, en Barcelona, el 20 de septiembre de 2019).
} 
funcionaban autónomamente como herramienta divulgativa de los ideales libertarios e instrumento educador de la clase obrera (Tavera, 1978). La publicación formaba parte de la empresa familiar de los Montseny: Joan Montseny (más conocido como Federico Urales), Teresa Mañé (Soledad Gustavo) y su hija Federica Montseny asumían las tareas de administración, dirección, coordinación y parte de la redacción de La Revista Blanca (Pradas, 2011).

El carácter intelectual y culturizador de esta publicación, junto a la profesionalización de su núcleo promotor, limitaban las posibilidades de participación de su público, formado por trabajadores y pequeñoburgueses afines al anarquismo y con un nivel de instrucción generalmente bajo (Pradas, 2011, p.153). No fue hasta los últimos años de su publicación que buscó la interacción con la audiencia. El principal mecanismo habilitado para este propósito fue el "Consultorio Sindical", que apareció a partir del noviembre de 1933 (número 252), y que recogía preguntas breves sobre esta temática enviadas por los lectores: constituía, pues, una herramienta de interacción bilateral. Rápidamente, a partir del número 254, esta sección se rebautizó con el nombre de "Consultorio General" y se amplió a política, historia, relaciones personales y salud. La sección se mantuvo hasta el final de la publicación de La Revista Blanca y se amplió de dos a cuatro páginas.

Por otra parte, a partir de febrero de 1935 apareció una nueva sección, también de interacción bilateral, llamada "Correo libre", que servía para responder las cartas dirigidas directamente a la redacción, fuera del consultorio. De algunas respuestas por parte de la revista se desprende que algunos lectores enviaban artículos y obras literarias - para La Revista Blanca o para las colecciones editoriales de la empresa-, que el medio desestimaba porque cuando los recibía ya no tenían actualidad o bien no presentaban la calidad suficiente para ser publicados. En algunos casos, sin embargo, se informaba que la dirección intentaría publicar los artículos de los lectores (números 345 y 353), lo que supondría una forma de interacción unilateral productiva, que no obstante fue anecdótica a lo largo de la trayectoria de la revista.

\subsection{API}


Si La Revista Blanca pudo esquivar la censura contra las ideas y publicaciones anarquistas porque no se trataba de un periódico sindical, sino divulgativo (Pradas, 2011), API permaneció la mayor parte de su vida en la clandestinidad estricta para evitar la represión franquista. El boletín, impulsado por el colectivo Agencia Popular Informativa, se planteaba como un producto periodístico que informaba de la actualidad de las acciones de la sociedad civil antifranquista (Pérez y Tébar, 2004, p.15), de manera que el grupo impulsor, formado por periodistas progresistas, no estaba adscrito a ningún partido o organización concreta para mantener la independencia y la profesionalidad y, al mismo tiempo, ser "un canal de expresión al movimiento obrero y popular en su totalidad" (API, 1975).

Las fuertes medidas de seguridad que tenía que tomar la agencia limitaban las oportunidades de practicar el periodismo participativo en cualquiera de sus vertientes. No existía sección de cartas de los lectores o de preguntas y respuestas, puesto que no se facilitaba ninguna dirección de contacto. Sin embargo, la participación se producía por otras vías: los partidos, sindicatos y otras organizaciones antifranquistas tenían acceso a la publicación mediante convocatorias, avisos y comunicación personal para que los periodistas informasen de asambleas, movilizaciones y otras acciones que habían llevado a cabo. Cada redactor de API disponía de su red de informantes con quienes mantenía un contacto regular (Vinyoles, Torns y Lanao, 1989, p.11). En algunos casos, la revista reproducía íntegra o parcialmente comunicados y documentos de organizaciones antifranquistas en sus artículos. En otros, eran directamente los colectivos quien firmaba los artículos - por ejemplo, la Comisión Coordinadora de Fuerzas Políticas de Cataluña, Comisiones Obreras o la Assemblea de Catalunya - o bien daban la réplica a un artículo aparecido en el boletín. Estos son casos de interacción unilateral productiva.

Pese a todo, la Agencia Popular Informativa pretendía que el medio fuera lo más participativo posible en la organización y toma de decisiones interna, pero también en la relación con los otros actores antifranquistas y los lectores: se quería que hubiera un "control crítico de colaboradores, corresponsales, lectores y organizaciones diversas del movimiento popular" y que este feedback se hiciera público "a fin de contribuir a 
depurar la función que tiene asignada API y la "contrainformación" en general" (API, 1975). Esta hubiera constituido una fórmula de interacción comunicativa impensable en los medios oficiales y autorizados por el régimen, que de todas maneras no se pudo llevar a cabo. Otro factor que dificultaba la interacción era la postura de las organizaciones antifranquistas, muchas de las cuales prefirieron centrar sus esfuerzos en publicar sus propios órganos en lugar de dar más apoyo a una publicación contrainformativa independiente (Pérez y Tébar, 2004).

\subsection{CRUÏLA - ILLACRUA}

Cruïlla -a partir del número 13 , Illacrua- se presentaba como un "medio de comunicación alternativo" y "participativo" (Illacrua, 1994, p.5) que sintonizaba "con los movimientos sociales anticapitalistas" (Illacrua, 2001, p.3). Pese a esta proximidad, la publicación siempre reiteró su autonomía y procuraba combinar la información sobre opciones sociales transformadoras con un tratamiento periodístico de calidad y la voluntad de llegar a un público amplio (Illacrua, 2002).

Desde el primer número, Cruïla - Illacrua incorporó varios mecanismos de participación. Al inicio, había secciones pensadas para que los lectores, tanto individuos como organizaciones, enviaran información: "Cartes", "El Far", "Comunicats" y "Bústia". A partir del número 137, la participación se reformuló con la aparición de una nueva sección, "Protesta... i viu!", donde los lectores enviaban informaciones sobre la actualidad y los movimientos sociales de sus poblaciones. A estas fórmulas de participación unilateral se sumaba otra: los artículos escritos por los lectores y colectivos sociales. En cada número había una o varias colaboraciones de este tipo, ya fueran propuestas por sus mismos autores o bien por encargo del equipo redactor de la revista para abordar un tema específico. En ambos casos, era frecuente que los textos se tuvieran que reelaborar para cumplir los criterios periodísticos y estilísticos de publicación (Jordi Garcia, 17/9/2019).

Con respecto a la interacción bilateral, se habilitaron algunos espacios, si bien no tuvieron la misma longevidad que aquellos destinados a la participación unilateral productiva. Entre diciembre de 1997 y junio de 2001, se encartó en la revista una tarjeta 
de suscripción que también incluía "El termòmetre d'lllacrua", pensada para que los lectores la llenaran y la enviaran indicando qué artículo les había gustado más, cuál menos y qué temas creían que se tenían que tratar más. A parte, la tarjeta ofrecía un espacio para que el lector apuntara información que consideraba interesante para la revista. A partir de 2006, se impulsó una nueva sección de participación bilateral, "La pregunta", que recogía las respuestas del público a un interrogante planteado por la revista.

La interacción comunicativa también fue presente desde los inicios. El manual de estilo se realizó a través de reuniones abiertas (Jordi Garcia, 17/9/2019). Una vez puesta en marcha, la revista impulsó un consejo asesor, al cual se invitaba a representantes de movimientos sociales y organizaciones civiles, así como subscritores y lectores. El órgano valoraba la trayectoria del medio, evaluaba su línea editorial y proponía temas nuevos o pedía que se reforzaran los que ya se reflejaban; otros medios alternativos precedentes, como el diario Liberación o la revista Bicicleta, habían contado con mecanismos similares (Jordi Garcia, 17/9/2019). Pero a medida que la revista se consolidaba y el colectivo de redactores ganaba peso, la participación en el consejo asesor fue menguando hasta desaparecer. Jordi Garcia valora que los movimientos sociales, al constatar la madurez del proyecto, no veían tan necesaria su implicación, aunque continuaban enviando informaciones. El final de la revista, cuando Illacrua se integró como sección de Directa, el año 2010, se condujo como un proceso participativo abierto a periodistas y subscritores de ambas cabeceras (Illacrua, 2009).

\subsection{DIRECTA}

Después de diez años publicándose bajo el paraguas de una asociación sin ánimo de lucro, el 2016 Directa devino una cooperativa de consumidores y usuarios. Esta modalidad de empresa concuerda especialmente con la ética participativa de los medios alternativos (Barranquero y Sánchez, 2018, p.48) y está siendo adoptada por algunos nuevos proyectos que apuestan por un periodismo de calidad y una relación más estrecha con la audiencia, como Crític (Canovaca, 2018). Así, esta transformación estructural ha implicado que los subscritores, que antes simplemente recibían el 
producto, tengan la opción de participar organizativamente en el desarrollo de Directa, de manera que se refuerza la construcción de una comunidad alrededor del medio (Rius, 2019). Desde Directa consideran que la revista "es mucho más que un producto" y que los lectores valoran de ella la voluntad de transformación social a través del periodismo (Ester Mora, 20/9/2019).

Directa contempla tres tipos de socios: los trabajadores, los colaboradores y los consumidores, que son la mayoría y que pueden ser tanto individuales como colectivos. El principal órgano de decisión es la Asamblea General, en la cual se convoca a todos los socios, además de estar abierta a subscritores y lectores, a pesar de que el derecho a voto solo lo pueden ejercer las personas socias del medio. Los otros espacios de participación, en los cuales intervienen representantes de los socios consumidores, son el Consejo Rector, que realiza el seguimiento del proyecto; el Consejo Social, que vela por que los nuevos colectivos que se adhieren a la cooperativa cumplan los requisitos estipulados en los estatutos, y el Consejo Periodístico, en el cual los consumidores aportan su visión y propuestas para el desarrollo informativo del proyecto (Ester Mora, 20/9/2019). Estos son espacios de interacción comunicativa, si bien la capacidad de decisión se limita a aquellos que sean socios de la cooperativa.

En cuanto a mecanismos de interacción bilateral, hasta el número 429 (abril de 2017), la revista publicaba en cada número un banner animando a los lectores a expresar, por correo electrónico, qué artículos les gustaban más y qué temas creían que había que tratan. Por otro lado, el medio facilita en su web, en la pestaña "Contacto", la dirección electrónica de cada sección de la revista. A través de este mecanismo, así como del teléfono y la comunicación personal con los periodistas, llegan propuestas de asuntos de actualidad a la redacción para que la revista los trate. La posibilidad de que los socios consumidores puedan enviar y publicar artículos hace que la frontera entre consumidores y colaboradores se difumine (Ester Mora, 20/9/2019); esta fluctuación entre ambas posiciones es una característica fundamental de los medios alternativos (Atton, 2002; Atton y Hamilton, 2008). Como en Illacrua, los textos deben tener un carácter periodístico y unos niveles mínimos de redactado, y son revisados y, si es necesario, adaptados por los periodistas. 
Directa se planteó, en los inicios del proyecto, un debate interno sobre si ofrecer la posibilidad de comentar las noticias de la edición en línea, mecanismo que se rechazó al constatar que en las secciones de comentarios de los cibermedios son frecuentes los insultos y las descalificaciones (Ester Mora, 20/9/2019). En cambio, respecto a la interacción bilateral, el medio procura contestar el feedback que recibe a través de sus redes sociales. Asimismo, el 2018 realizó una encuesta entre los subscritores -con 200 respuestas de un total de 2.700 subscripciones - para conocer como valoraban la atención, la calidad de la revista y el tipo de contenidos que se estaban publicando.

Tabla 2. Resumen de resultados. Mecanismos de participación en casos analizados.

\begin{tabular}{|c|c|c|c|c|}
\hline $\begin{array}{c}\text { Tipo de } \\
\text { interacción }\end{array}$ & La Revista Blanca & $A P I$ & Cruïla-Illacrua & Directa \\
\hline Unilateral & $\begin{array}{l}\text { Contenidos } \\
\text { enviados por los } \\
\text { lectores } \\
\text { (puntual). }\end{array}$ & $\begin{array}{l}\text { Contenidos } \\
\text { enviados por } \\
\text { los lectores. }\end{array}$ & $\begin{array}{l}\text { Contenidos } \\
\text { enviados por los } \\
\text { lectores. } \\
\text { Cartas al director } \\
\text { o a los redactores. }\end{array}$ & $\begin{array}{l}\text { Contenidos } \\
\text { enviados por los } \\
\text { lectores. }\end{array}$ \\
\hline Bilateral & $\begin{array}{l}\text { Sección de } \\
\text { preguntas y } \\
\text { respuestas. } \\
\text { Correspondencia } \\
\text { con la redacción } \\
\text { (puntual). }\end{array}$ & & $\begin{array}{l}\text { Encuestas y } \\
\text { votaciones. } \\
\text { Sección de } \\
\text { preguntas y } \\
\text { respuestas. } \\
\text { Correspondencia } \\
\text { con la redacción. }\end{array}$ & $\begin{array}{l}\text { Encuestas y } \\
\text { votaciones. } \\
\text { Correspondencia } \\
\text { con la redacción. }\end{array}$ \\
\hline Comunicativa & & & $\begin{array}{l}\text { Correspondencia } \\
\text { con la redacción. } \\
\text { Actos públicos, } \\
\text { fórums y otros } \\
\text { espacios de } \\
\text { debate. } \\
\text { Reuniones y } \\
\text { encuentros con } \\
\text { el director o los } \\
\text { redactores. }\end{array}$ & $\begin{array}{l}\text { Correspondencia } \\
\text { con la redacción. } \\
\text { Actos públicos, } \\
\text { fórums y otros } \\
\text { espacios de } \\
\text { debate. } \\
\text { Reuniones y } \\
\text { encuentros con } \\
\text { el director o los } \\
\text { redactores } \\
\text { (participación en } \\
\text { los órganos } \\
\text { decisorios de la } \\
\text { cooperativa). }\end{array}$ \\
\hline
\end{tabular}


Fuente: Elaboración propia.

\section{CONCLUSIONES}

Los resultados obtenidos en el estudio planteado en este artículo confirman la hipótesis de partida: en la historia de la prensa alternativa catalana ha existido una variedad de enfoques de la participación, que han resultado en diversas oportunidades estructurales para la interacción con los públicos. Así, las cabeceras estudiadas han promovido la emergencia de audiencias activas a partir de implicarlas en los procesos de producción de contenidos y toma de decisiones. Comprender con perspectiva histórica la ética y los mecanismos participativos de la comunicación alternativa supone asumir que no existe una única historia de la participación en los medios, sino múltiples, y que no se les puede aplicar automáticamente un esquema de progreso lineal (Carpentier, Dahlgren y Pasquali, 2014).

La Revista Blanca constituye un caso de lo que Traber llama advocacy media (1985, citado en Atton, 2002): es crítica respecto a los medios de comunicación dominantes, pero organizativamente es vertical y poco permeable a la participación. Las limitaciones impuestas por la dictadura franquista dificultaron que $A P I$ ofreciera efectivamente fórmulas de participación bilateral y comunicativa, aunque la proximidad con partidos y organizaciones opositoras propiciara una participación soterrada. Cruïla - Illacrua combina rasgos de los modelos crítico y radical: utiliza algunas estrategias próximas a las de los medios convencionales, pero se mantiene atento a los movimientos sociales y se abre a la participación a través de múltiples vías. En Directa, los socios consumidores participan de la toma de decisiones y del seguimiento del proyecto, al mismo tiempo que contempla que socios, subscritores y lectores envíen y publiquen informaciones, aunque el peso de la redacción recae en trabajadores y colaboradores. En estos dos últimos casos, los medios incluyen mecanismos de los tres tipos descritos por Schultz (2000) y de las modalidades participativa y productiva (Masip y Suau, 2014).

Los resultados de este estudio refuerzan las consideraciones de Atton sobre la interacción entre emisores y receptores en los medios alternativos: se permite una participación en la creación, producción y diseminación más amplia que en los medios 
de comunicación masivos (Atton, 2002). Mientras que la mayoría de los medios convencionales han orientado la participación hacia finalidades comerciales (Paulussen, Heinonen, Domingo y Quandt, 2007), el rechazo de una concepción mercantilista del hecho periodístico en los medios alternativos posibilita la aparición de un "proyecto informativo común" que propicia una participación "con finalidad desde el punto de vista de cultura democrática" (Masip, Guallar, Peralta, Ruiz y Suau, 2015, p.255). En este sentido, apunta Masip, es entre los nuevos medios que operan fuera del mainstream donde se producen las iniciativas más interesantes, basadas "en una estrecha relación de reconocimiento mutuo entre los periodistas y su audiencia" (Masip, 2016, p.328). Esta dinámica se da especialmente en los medios cooperativistas, los cuales Barranquero y Sánchez (2018) identifican como un modelo derivado de la comunicación alternativa.

Sin embargo, el estudio realizado presenta limitaciones evidentes, principalmente un nombre muy reducido de casos - aunque se ha procurado que fueran lo más significativos posible- que no permite llegar a conclusiones más generales o extrapolar automáticamente los resultados al conjunto de la prensa alternativa. Sería necesario incluir en futuros análisis otros enfoques entre los medios alternativos - como los comunitarios y ciudadanos - para evaluar sus fórmulas de implicación de la ciudadanía, así como establecer comparaciones con los medios convencionales. En paralelo, es necesario avanzar en los estudios de audiencias de los medios alternativos para que sus públicos dejen de ser, en palabras de Downing, "virtualmente desconocidos" (2003).

Sobre el objetivo secundario que hemos planteado, se ha esbozado una cronología histórica de la prensa alternativa en el ecosistema comunicativo de Cataluña. Como apuntan Atton y Hamilton (2008) y Forde (2011), los medios alternativos han existido desde los inicios de la modernidad y se han construido constantemente en una relación dialéctica con las formas dominantes del periodismo. Reseguir y analizar su desarrollo permite constatar la diversidad del fenómeno periodístico en todas sus vertientes producción de contenidos, organización y relación con la audiencia- a lo largo del tiempo. De esta manera, se obtendría una visión de conjunto más compleja de la historia de la comunicación mediática, que incluya también a las audiencias y sus 
comportamientos. En este sentido, consideramos que el presente estudio constituye una contribución preliminar para una historia de la prensa alternativa catalana, que puede ser útil para investigaciones más exhaustivas que enriquezcan la tradición de la investigación en periodismo y comunicación.

\section{REFERENCIAS BIBLIOGRÁFICAS}

Atton, C. (2002). Alternative Media. SAGE: Londres

Atton, C., y Hamilton, J. (2008). Alternative Journalism. Londres: SAGE Publications

Bailey, O. G., Cammaert, B., y Carpentier, N. (2007). Understanding alternative media. Regne Unit: McGraw-Hill Education.

Barnes, R. (2014). The «ecology of participation». A study of audience engagement on alternative journalism websites. Digital journalism, 2(4), 542-557. Recuperado de https://www.tandfonline.com/doi/abs/10.1080/21670811.2013.859863

Barranquero, A., y Meda, M. (2015). Los medios comunitarios y alternativos en el ciclo de protestas ciudadanas desde el 15M. Athenea Digital, 15 (1), 139-170. Recuperado de https://ddd.uab.cat/pub/athdig/athdig_a2015v15n1/athdig_a2015v15n1p139.pdf

Barranquero, A., y Sánchez, M. (2018). Cooperativas de medios en España. Un periodismo emprendedor y ciudadano en tiempos de crisis. REVESCO: Revista de estudios cooperativos, (128), 36-58. Recuperado de https://dialnet.unirioja.es/servlet/articulo?codigo $=6524644$

Canovaca, E. (2018). Nuevos modelos de negocio en la prensa digital: el caso de Crític. Revista de la Asociación Española de Investigación de la Comunicación, 5(9), 33-40. Recuperado de http://revistaeic.eu/index.php/raeic/article/view/150/142

Carpentier, N., Dahlgren, P., y Pasquali, F. (2014). The democratic (media) revolution: A parallel history of political and media participation. En A Carpentier, N., Schrøder, K. y Hallet, L. Audience transformations. Shifting audience positions in late modernity. Nueva York: Routledge. 
Cruz-Álvarez, J., y Suárez-Villegas, J. C. (2012). Ética de la participación ciudadana en los discursos periodísticos digitales. El profesional de la información, 21(4), 375-380. Recuperado de http://www.elprofesionaldelainformacion.com/contenidos/2012/julio/07.pdf

Downing, J. (2001). Radical Media: Rebellious Communication and Social Movements. Sage: Londres.

Downing, J. (2003). Audiences and readers of alternative media: The absent lure of the virtually unknown. Media, Culture \& Society, 25(5), 625-645. Recuperado de https://journals.sagepub.com/doi/pdf/10.1177/01634437030255004

Dalmases, P. I. (1992). La prensa anarcosindicalista en Cataluña 1.835-1.939. (n.p.): Asociación Española de Periodistas, Corresponsales y Colaboradores.

Fleischman, L., Reguero, N., y Sáez, C. (2009). Políticas de comunicación y sustentabilidad del tercer sector de la comunicación: el caso catalán en el contexto español y europeo. A Actas del VII Congreso Internacional de ULEPICC. Universidad Carlos III. Madrid. Recuperado de http://www.medioscomunitarios.net/barcelona/IMG/pdf/Ulepicc09 TSC VF2.pdf

Forde, S. (2011). Challenging the News: the Journalism of Alternative and Community Media. (n.p.): Macmillan International Higher Education.

Fuchs, C. (2010). Alternative Media as Critical Media. European Journal of Social Theory, 13(2), 173-192. Recuperado de https://journals.sagepub.com/doi/abs/10.1177/1368431010362294

Gómez-Mompart, J. L. (1992). La gènesi de la premsa de masses a Catalunya (19021923). Barcelona: Pòrtic.

González, R. (2011). Xarxes crítiques i polítiques públiques: els impactes del moviment per l'okupació a Catalunya y Madrid (1984-2009). (Tesi doctoral). Cerdanyola del Vallès: Universitat Autònoma de Barcelona. Recuperado de https://ddd.uab.cat/record/127278 
Griffen-Foley, B. (2004). From Tit-Bits to Big Brother: A century of audience participation in the media. Media, Culture \& Society, 26(4), 533-548. Recuperado de https://journals.sagepub.com/doi/abs/10.1177/0163443704044216

Guillamet, J. (1983). La premsa comarcal: un model català de periodisme popular. Departament de Cultura de la Generalitat de Catalunya: Barcelona.

Guillamet, J. (2003). Estat de la investigació en història de la premsa i del periodisme a Catalunya. Treballs de Comunicació, 18, 145-163. Recuperado de https://www.raco.cat/index.php/TreballsComunicacio/article/viewFile/242880/32554 $\underline{9}$

Hamilton, J. (2000). Alternative media: Conceptual difficulties, critical possibilities. Journal of Communication Inquiry, 24(4), 357-378.

https://doi.org/10.1177/1464884910385191

Harcup, T. (2011). Alternative journalism as active citizenship. Journalism, 12(1), 15-31. https://doi.org/10.1177\%2F1464884910385191

Jeppesen, S. (2016). Understanding Alternative Media Power: Mapping Content \& Practice to Theory, Ideology, and Political Action. Democratic Communiqué, 27(1), 54. Recuperado de https://journals.flvc.org/demcom/article/view/88322

López, M. (1994). Un periodisme alternatiu i autogestionari. Barcelona: Col·legi de Periodistes de Catalunya.

López-García, X. (2007). Gestión de las vías de participación en el ciberperiodismo. Estudios sobre el mensaje periodístico, 13, 111-121. Recuperado de https://www.researchgate.net/profile/Xose Garcia/publication/27593719 Gestion d e las vias de participacion_en el ciberperiodismo/links/0c96052f2aad65040300000 $\underline{0 . p d f}$

Maristany, G., y Musons, A. (2002). Del desencant a la contrainformació. La premsa de barris a Barcelona. 1976-2001. Barcelona: Col·legi de Periodistes de Catalunya. 
Masip, P. (2016). Investigar el periodismo desde la perspectiva de las audiencias. El profesional de la información (EPI), 25(3), 323-330.

https://doi.org/10.3145/epi.2016.may.01

Masip, P., y Suau, J. (2014). Audiencias activas y modelos de participación en los medios de comunicación españoles. Hipertext.net: Revista Académica sobre Documentación Digital y Comunicación Interactiva, (12). Recuperado de https://www.raco.cat/index.php/Hipertext/article/view/274308

Masip, P., Guallar, J., Peralta, M., Ruiz, C., y Suau, J. (2015). Audiencias activas y periodismo: ¿ciudadanos implicados o consumidores motivados?. Brazilian Journalism Research, 11(1), 240-261. Recuperado de https://bjr.sbpjor.org.br/bjr/article/view/795 Mauri-Ríos, M., y Ramon-Vegas, X. (2015). Nuevos sistemas de rendición de cuentas de la información periodística. Exploración del escenario online español. El profesional de la información, 24(4), 380-389. https://doi.org/10.3145/epi.2015.jul.04

Mejía, J. (2000). El muestreo en la investigación cualitativa. Investigaciones Sociales, 5 , 165-180. Recuperado de https://revistasinvestigacion.unmsm.edu.pe/index.php/sociales/article/view/6851 Moragas, M., y Prado, E. (2002). Les televisions locals a Catalunya: de les experiències comunitàries a les estratègies de proximitat. Quaderns del CAC, número extraordinario. Recuperado de http://www.mesadiversitat.cat/pfw files/cma/recerca/quaderns cac/tvlocal.pdf Paulussen, S., Heinonen, A., Domingo, D., y Quandt, T. (2007). Doing it together: Citizen participation in the professional news making process. OBSERVATORIO (OBS*), 1(3), 131-154. Recuperado de https://biblio.ugent.be/publication/387947/file/6798667

Pérez, J., y Tébar, J. (2004). Apuntes para el estudio de una experiencia contrainformativa durante el tardofranquismo: la «Agencia Popular Informativa», 1972-1976. En La transición a la democracia en España: actas de las VI Jornadas de 
Castilla-La Mancha sobre Investigación en Archivos: Guadalajara, 4-7 de noviembre 2003. Asociación de Amigos del Archivo Histórico Provincial de Castilla La Mancha. Recuperado de http://biblioteca2.uclm.es/biblioteca/ceclm/websCECLM/transici\%F3n/PDF/0105.\%20Texto.pdf

Pradas, M. A. (2011). La Revista Blanca. Origen, auge i decadència d'una publicació filollibertària barcelonina (1923-1936). Barcelona: Col·legi de Periodistes y Diputació de Barcelona

Prado, E. (1980). El desenvolupament de les ràdios lliures a Espanya. Anàlisi: quaderns de comunicació y cultura, 1, 155-167. Recuperado de http://www.raco.cat/index.php/Analisi/article/view/40962

Rius, J. C. (2019). Periodisme de comunitat. El repte de servir els lectors en l'era de les xarxes y no morir en l'intent. Capçalera, 180, 12-17.

Sáez, C. (2009). Tercer sector de la comunicación teoría y praxis de la televisión alternativa: una mirada a los casos de España, Estados Unidos y Venezuela (tesi doctoral). Barcelona: Universitat Autònoma de Barcelona. Recuperado de https://ddd.uab.cat/record/55187

Sandoval, M. (2009). A critical contribution to the foundations of alternative media studies. Kurgu: Online International Journal of Communication Studies, 1. Recuperado de http://openaccess.city.ac.uk/id/eprint/14324/

Sandoval, M., y Fuchs, C. (2010). Towards a critical theory of alternative media. Telematics and Informatics, 27(2), 141-150. Recuperado de https://www.sciencedirect.com/science/article/pii/S0736585309000410

Schultz, T. (2000). Mass media and the concept of interactivity: an exploratory study of online forums and reader email. Media, culture \& society, 22(2), 205-221. https://doi.org/10.1177/016344300022002005 
Tamarit, A. (2012). Las radios comunitarias: entre el compromiso y el espejismo de lo alternativo. A Martínez, M., Mayugo, C. y Tamarit, A. (coords.). Comunidad y Comunicación. Prácticas comunicativas y medios comunitarios en Europa y América Latina. Madrid: Fragua.

Tavera, S. (1978). La premsa anarco-sindicalista (1868-1931). Recerques: història, economia, cultura, 8, 85-102. Recuperado de https://www.raco.cat/index.php/Recerques/article/viewFile/137554/241347 Tavera, S. (1992). Solidaridad Obrera. El fer-se i desfer-se d'un diari anarco-sindicalista (1915-1939). Barcelona: Col·legi de Periodistes de Catalunya y Diputació de Barcelona. Tresserras, J. M. (2000). Els sistemes de comunicació i cultura a Catalunya durant el segle XX. Una proposta d'interpretació. Cercles. Revista d'Història Cultural, 3, 59-73. Recuperado de http://revistes.ub.edu/index.php/cercles/article/view/16692 Tubau, F., y Vallès, J. (1999). Emili Prado: «La ràdio municipal ha de recuperar la capacitat dinamitzadora que es buscava en el seu origen». Antena local, 9, 14-15.

Vatikiotis, P. (2005). Communication Theory and Alternative Media. Westminster Papers in Communication and Culture, 2(1). Recuperado de https://www.researchgate.net/publication/253989689 Communication Theory and Alternative Media

Vinyoles, C., Torns, M. y Lanao, P. (1989). API, Agència Popular Informativa, 1972-1976. Capçalera, 4, 10-15. Recuperado de https://www.raco.cat/index.php/Capcalera/article/viewFile/322780/413407 